\title{
Utility of Tracing as a Memory Storage Method
}

\author{
Makana Tsutsui', Masako Notoya², Daisuke Kimura3 ${ }^{3}$, Ken Nakatani' ${ }^{3}$, Takashi Fujita ${ }^{4}$, \\ Nobuyuki Sunahara ${ }^{5}$, Katsumi Inoue ${ }^{5}$
}

\author{
${ }^{1}$ Japanese Red Cross Kanazawa Hospital, Ishikawa, Japan \\ ${ }^{2}$ Health Medical Department, Kytogakuen University, Kyoto, Japan \\ ${ }^{3}$ Department of Rehabilitation Sciences, Faculty of Allied Health Sciences, Kansai University of Welfare Sciences, Osaka, Japan \\ ${ }^{4}$ Division of Occupational Therapy, Faculty of Care and Rehabilitation, Seijoh University, Aichi, Japan \\ ${ }^{5}$ School of Health Sciences, College of Medical, Pharmaceutical and Health Sciences, Kanazawa University, Ishikawa, Japan \\ Email: t.makana.0321@gmail.com, notoya@kyotogakuen.ac.jp,dkimura@tamateyama.ac.jp, knakatani@tamateyama.ac.jp, \\ takafuji120@yahoo.co.jp, sunahara@mhs.mp.kanazawa-u.ac.j, tsubura1@staff.kanazawa-u.ac.jp
}

How to cite this paper: Tsutsui, M., Notoya, M., Kimura, D., Nakatani, K., Fujita, T., Sunahara, N. and Inoue, K. (2017) Utility of Tracing as a Memory Storage Method. World Journal of Neuroscience, 7 , 216-222.

https://doi.org/10.4236/wjns.2017.72017

Received: February 28, 2017

Accepted: May 7, 2017

Published: May 10, 2017

Copyright () 2017 by authors and Scientific Research Publishing Inc. This work is licensed under the Creative Commons Attribution International License (CC BY 4.0).

http://creativecommons.org/licenses/by/4.0/

\begin{abstract}
Although many studies have explored the utility of tracing as a rehabilitation approach for patients with aphasia and alexia and for Japanese patients with various disabilities, this may be the first study to demonstrate the superiority of tracing over copying for enhancing long-term memory. We investigated the utility of tracing as a memory storage method. Young and elderly participants learned a figure from the Rey-Osterrieth complex figure test by copying or tracing its outline. They were asked to reproduce the figure after $3 \mathrm{~min}$ and 3 days. Although the copying group performed better than the tracing group in immediate recall after $3 \mathrm{~min}$, the performance of the tracing and copying groups after 3 days was similar. Among younger participants, the tracing group achieved higher scores than the copying group after 3 days; however, the difference was not statistically significant. Copying as a learning strategy has a substantial temporal gradient of memory loss; tracing may be more appropriate for improving long-term memory. This result could have considerable practical usefulness, e.g., among professionals who provide memory training for the elderly. Tracing, which uses visuomotor memory, is acquired earlier than transcription. Tracing may be effective for rehabilitation because it is a developmentally appropriate approach to early instruction.
\end{abstract}

\section{Keywords}

Visual Memory, Learning, Long-Term Memory

\section{Introduction}

Most of our daily activities are supported by a complex cognitive system known as memory. Squire and Zolz-Morgan [1] classified memories into two categories: 
declarative (or explicit, involving specific facts and events) and non-declarative (or implicit, involving unconscious recollections, such as visuomotor skills and habits). Numerous examination methods have been developed to evaluate the different types of memories in clinical environments.

Visuomotor memory, which connects visual information with physical movements to enable people to perform daily functions such as cooking or driving a car, is neurologically controlled by the basal ganglia. Neurological studies of child development have reported that tracing, which uses visuomotor memory, is acquired earlier than transcription [2]. Tracing may also be effective for rehabilitation because it is a developmentally appropriate approach to early instruction.

Several studies have reported on the use of tracing as a rehabilitation approach for patients with aphasia, alexia, and agraphia or those who have had to change their writing hand after suffering a stroke [3] [4] [5]. However, little, if any, focus has been placed on tracing as a rehabilitation approach for people with memory disorders. However, differences in memory reproduction due to tracing and copying have not been mentioned in previous studies. In order to prove that tracing is effective for rehabilitation, it is necessary to verify the difference in memory reproduction by tracing and copying. First of all, it is necessary to demonstrate and verify healthy subjects for that purpose.

In this study, we sought to examine the effectiveness of tracing in comparison with copying as a memorization technique. We achieved this objective by asking participants to either trace or copy figures and then by evaluating their ability to remember and sketch the same figure both after $3 \mathrm{~min}$ and 3 days.

\section{Materials and Methods}

\section{Participants and Procedures}

The participants were 28 healthy students attending the Kanazawa University in Japan and 28 elderly members of an urban social club. All participants had no known health problems and gave informed consent for their participation. The elderly participants were evaluated using the Mini-Mental State Examination (MMSE) to confirm that they could achieve a score of 24 or higher (indicating the absence of dementia). All of the young participants were the students in Kanazawa University. As all young participants are healthy and have no trouble with academic work, MMSE for young group was omitted. The experiment was conducted with the approval of the medical ethics committee of Kanazawa University.

Each age cohort was divided into the copying and tracing groups. We don't have training for the participants, only have the memory test. Table 1 indicates the average age of each group and the average MMSE score of the elderly participants.

Participants were shown a figure from the Rey-Osterrieth complex figure test (ROCF), which is commonly used as a test for neurological status or cognitive development. Each participant in the copying group was given a separate sheet 
Table 1. Age and MMSE scores of participants.

\begin{tabular}{cccc}
\hline & & Mean age \pm SD & MMSE \pm SD \\
\hline \multirow{2}{*}{ Elderly } & Copying & $73 \pm 6.8$ & $28.4 \pm 1.6$ \\
& Tracing & $75.8 \pm 5.9$ & $28.2 \pm 1.9$ \\
\multirow{2}{*}{ Young } & Copying & $21.8 \pm 0.4$ & No data \\
& Tracing & $20.5 \pm 0.5$ & No data \\
\hline
\end{tabular}

of paper and asked to copy a figure from ROCF onto that sheet. The figure is placed so that its length runs along the subject's horizontal plane. After the participant had completed copying the figure, interference simulation (talking about issues unrelated to the task) took place for $3 \mathrm{~min}$; thereafter, the participant was asked to draw the figure again as an immediate recall test, without referring to the original or copy. After 3 days, as a delayed recall test, the participants were instructed to recall and draw the figure.

Participants in the tracing group followed the same procedures, except that the figure was directly traced onto the model of the ROCF sheet. To highlight their tracing, the participants were instructed to use a red pencil. No tracing order was determined, i.e., the participants were free to trace the lines in any order.

Both copying and tracing performed only once.

The participants' drawings were evaluated using a conventional 36-point rating scale. Their scores were worked out by assessment of EM Taylor [6]. Although several scoring systems have been published, the most commonly used continuous to be the Rey-O/Taylor/MCG unit scoring method which divides the figures into 18 score-able units. Since a correctly placed and proportional copy of each unit earns 2 points, the highest possible score is 36 .

For statistical analysis, two-way ANOVA was performed to compare the scores of the copying and tracing groups $3 \mathrm{~min}$ and 3 days after the experiment (Table 2). When interactions were observed, the simple main effect was examined using the t-test (Table 3 and Table 4).

\section{Results}

With regard to the young participants (Figure 1), on the immediate recall test (reproduction after $3 \mathrm{~min}$ ), the copying group achieved significantly higher scores than the tracing group. However, after 3 days, the tracing group outperformed the copying group; however, the difference was not statistically significant. When the two recall tests were compared within each group, the copying group had significantly lower scores after 3 days than after 3 min; however, the tracing group had no significant difference between its immediate recall and delayed recall scores.

Among the elderly participants (Figure 2), the copying group scored slightly higher after $3 \mathrm{~min}$; however, the difference in scores between the two groups was not statistically significant at either time point. However, the copying group had a significantly lower score after 3 days than after $3 \mathrm{~min}$, and no significant di- 


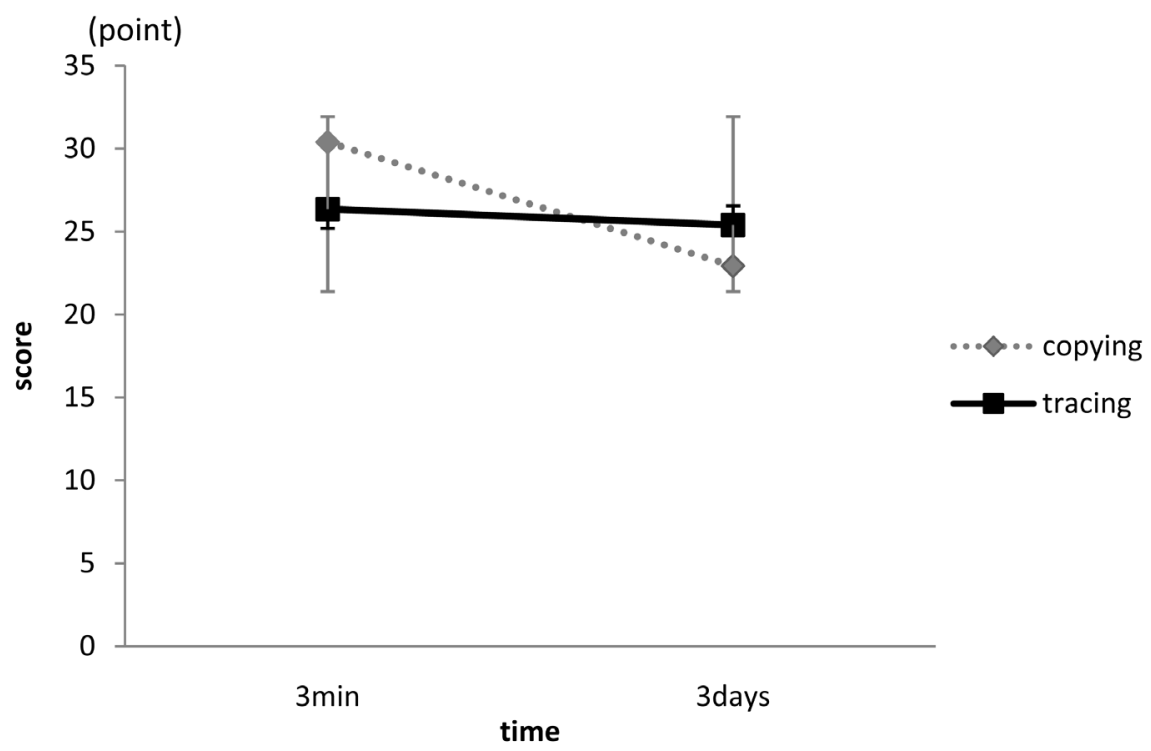

Figure 1. Comparison of copying and tracing in healthy young participants.

Table 2. Results of two-way ANOVA. (a) Young participants; (b) Elderly participants.

(a)

\begin{tabular}{cccccc}
\hline & Sum of square & Degree of freedom & Mean of square & F-value & $P$-value \\
\hline Time & 347.504 & 1 & 347.504 & 26.085 & $<0.001^{* *}$ \\
Time $\times$ Method & 250.754 & 1 & 250.754 & 18.823 & $<0.001^{* *}$ \\
Error & 346.366 & 26 & 13.322 & & \\
Method & 2.290 & 1 & 29.290 & 0.975 & 0.332 \\
Error & 780.723 & 26 & 30.328 & 30.028 & \\
\hline
\end{tabular}

(b)

\begin{tabular}{cccccc}
\hline & Sum of square & Degree of freedom & Mean of square & F-value & $P$-value \\
\hline Time & 42.875 & 1 & 42.875 & 15.174 & $<0.001^{* *}$ \\
Time $\times$ Method & 27.161 & 1 & 27.161 & 9.613 & $<0.005^{* *}$ \\
Error & 73.464 & 26 & 2.826 & & \\
Method & 27.161 & 1 & 27.161 & 0.369 & 0.549 \\
Error & 1915.464 & 26 & 73.672 & & \\
\hline
\end{tabular}

Table 3. Paired $t$-test.

\begin{tabular}{ccccccc}
\hline Participants & & Tracing & Copying & Tratio & Degree of freedom & $p$ value \\
\hline \multirow{2}{*}{ Yong } & 3 min & $27.61 \pm 4.52$ & $33.29 \pm 2.30$ & -4.192 & 26 & $<0.001^{\star *}$ \\
& 3 days & $26.86 \pm 5.45$ & $24.07 \pm 5.59$ & 1.334 & 25.982 & 0.194 \\
\multirow{2}{*}{ Elderly } & 3 min & $18.36 \pm 7.49$ & $21.14 \pm 4.77$ & -1.173 & 26 & 0.251 \\
& 3 days & $18.00 \pm 7.49$ & $18.00 \pm 4.24$ & 0.000 & 26 & 1.000 \\
\hline
\end{tabular}




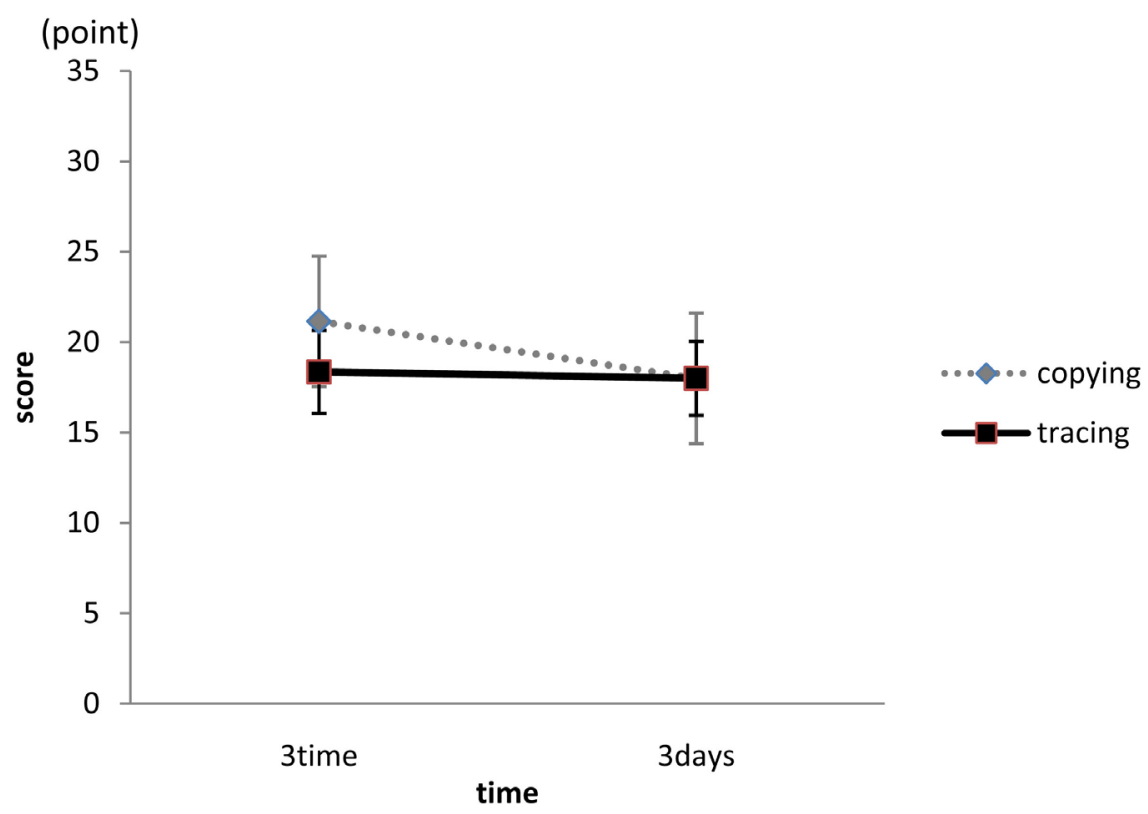

Figure 2. Comparison of copying and tracing in healthy elderly participants.

Table 4. Student t-test.

\begin{tabular}{ccccccc}
\hline Participants & & $3 \mathrm{~min}$ & 3 days & Tratio & Degree of freedom & $p$ value \\
\hline \multirow{2}{*}{ Yong } & Tracing & $27.61 \pm 4.52$ & $26.86 \pm 5.45$ & 5.178 & 13 & $<0.000^{* *}$ \\
& Copying & $33.29 \pm 2.30$ & $24.07 \pm 5.59$ & 1.273 & 13 & 0.225 \\
\multirow{2}{*}{ Elderly } & Tracing & $18.36 \pm 7.49$ & $18.00 \pm 7.49$ & 5.925 & 13 & $<0.000^{* *}$ \\
& Copying & $21.14 \pm 4.77$ & $18.00 \pm 4.24$ & 0.492 & 13 & 0.631 \\
\hline
\end{tabular}

${ }^{* *} p<0.001$

fference was observed between the immediate recall and delayed recall scores for the tracing group.

\section{Discussion}

We assessed the results of copying and tracing performed by healthy young and elderly participants to examine the usefulness of tracing in promoting memory. Although the copying group performed better than the tracing group in immediate recall after $3 \mathrm{~min}$ (with a significant difference in scores among younger participants), the performance of the tracing and copying groups after 3 days was similar. Among the younger participants, the tracing group achieved higher scores than the copying group after 3 days; however, the difference was not statistically significant.

These results indicated a difference in the temporal gradient of memory loss between the copying and tracing groups. The participants who learned the figure by copying it achieved high scores when asked to reproduce the figure immediately after $3 \mathrm{~min}$; however, their memory degraded significantly over 3 days. In contrast, the memory storage attained through tracing did not decrease substantially, suggesting that tracing is an appropriate method for improving long-term 
memory. Furthermore, in this study, the improvement was found especially in the young group. A prior study on learning to draw indicated that the copying group achieved a higher score when asked to reproduce a figure after a short period; however, no significant score difference was observed after a long period [7]; these results are consistent with those of this study.

The task of reproduction by copying requires not only a sufficient capacity of visual memory but also visual constructional and spatial abilities. Although healthy young people have these abilities, the elderly are often weak in these areas. However, the task of tracing does not require the abovementioned visual constructional and spatial abilities. The skills used in tracing involve the somatosensory system.

These considerations and the study results suggested that although copying activities can be used in a rehabilitation program to improve the reduced visual constructional and spatial abilities of the elderly, tracing can be introduced as a novel method for memory rehabilitation of the elderly to evoke memories using the somatosensory system.

In this study, the elderly in the tracing group scored lower than those in the copying group on the immediate recall test; however, the difference was not significant. Therefore, it may seem that copying has some benefit, particularly in inducing short-term memory, and we plan to introduce the task of tracing in combination with copying in their memory training.

Although some training programs designed to teach writing skills to infants have used tracing as an instructional method, to the best of our knowledge, no studies have been conducted on memory training for the elderly that incorporates both copying and tracing. We intend to implement and evaluate the effectiveness of such a program for the elderly in our future research.

\section{Conclusion}

This study investigated the utility of tracing as a long-termmemory storage method. A preliminary study was conducted to examine the effectiveness of tracing in comparison with copying as a memorization technique, with participants either copying or tracing a figure from ROCF. The findings indicated that copying as a learning strategy has a substantial temporal gradient of memory loss and that tracing may be a more appropriate method for improving long-term memory. In future, we conduct to brain-damaged patients' memory rehabilitation.

\section{Acknowledgements}

We would like to thank the students from Kanazawa University and the elderly members of the urban social club for their participation and cooperation.

\section{References}

[1] Squire, L.R. and Zolz-Morgan, S. (1988) Memory: Brain System and Behavior. Trends in Neuroscience, 11, 170-175. https://doi.org/10.1016/0166-2236(88)90144-0

[2] Watanabe, M. (2010) Writing and Written Language Acquisition Levels in Mentally 
Disabled Children, in Relationship to Mental Age. The Japanese Journal of Developmental Psychology, 21, 169-181.

[3] Akezaki, Y., Kawakami, Y., Hiraga, Y., Nomura, T. and Sato, A. (2009)An Exercise Method to Writing Accuracy of the Nondominant Hand-The Utility of the Schreibendes Writing Exercise. Rigakuryoho Kagaku, 24, 689-692.

https://doi.org/10.1589/rika.24.689

[4] Endo, K., Suzuki, K., Hirayama, K., Fujii, T., Kumabe, T. and Mori, E. (2010) Meaning, Phonological, Orthography and Kinestic Route of Reading and Writing: A Case with Alexia and Agraphia Due to the Left Parietal Lesion. Brain Nerve, 62 , 991-996.

[5] Fukunaga, S., Abe, H., Hattori, F., Kobayashi, H. and Tsuzuki, S. (1998) Effect of Pseudonym tracing Reading in Aphasic. General Rehab, 26, 371-376.

[6] Lezak, M.D., Howieson, D.B. and Tranel, D. (2012) Chap14 Construction and Motor Performance. Neuropsychological Assessment, 5th Edition, 580.

[7] Gonzalez, C., Anderson, J., Culmer, P., Burke, M.R., Mon-Willams, M. and Wilkie, R.M. (2011) Is Tracing or Copying Better When Learning to Reproduce a Pattern? Experimental Brain Research, 208, 459-465.

https://doi.org/10.1007/s00221-010-2482-1

Submit or recommend next manuscript to SCIRP and we will provide best service for you:

Accepting pre-submission inquiries through Email, Facebook, LinkedIn, Twitter, etc. A wide selection of journals (inclusive of 9 subjects, more than 200 journals) Providing 24-hour high-quality service User-friendly online submission system Fair and swift peer-review system Efficient typesetting and proofreading procedure Display of the result of downloads and visits, as well as the number of cited articles Maximum dissemination of your research work

Submit your manuscript at: http://papersubmission.scirp.org/

Or contactwjns@scirp.org 